\title{
Filigrane
}

Écoutes psychothérapiques

\section{La psychanalyse : parcours d'une nomade}

\section{Gisèle Lacerte}

Volume 17, numéro 1, printemps 2008

L’avenir du clinicien I

URI : https://id.erudit.org/iderudit/018791ar

DOI : https://doi.org/10.7202/018791ar

Aller au sommaire du numéro

Éditeur(s)

Revue Santé mentale au Québec

ISSN

1192-1412 (imprimé)

1911-4656 (numérique)

Découvrir la revue

Citer cet article

Lacerte, G. (2008). La psychanalyse : parcours d'une nomade. Filigrane, 17(1), 95-104. https://doi.org/10.7202/018791ar
Résumé de l'article

L'événement tragique - l'événement psychique — par l'excès qui le caractérise cherche à déverser son excédent. Son mouvement trace la trajectoire de la vie où s'entrelacent les expériences personnelles et professionnelles. L'auteur propose ici son propre parcours, elle retrace le long chemin qui l'a menée, depuis les exils obligés jusqu'au nomadisme de la vie adulte, à s'inscrire à la Société de psychanalyse de Montréal. L'inoubliable des amours perdues détermine la poursuite inlassable des rencontres. C'est cette quête, vouée à la répétition, que l'auteur poursuit dans son travail de clinicienne et dans les aspects théoriques qu'il comporte. d'utilisation que vous pouvez consulter en ligne.

https://apropos.erudit.org/fr/usagers/politique-dutilisation/ 


\title{
La psychanalyse: parcours d'une nomade
}

\author{
gisèle lacerte
}

L'événement tragique - l'événement psychique - par l'excès qui le caractérise cherche à déverser son excédent. Son mouvement trace la trajectoire de la vie où s'entrelacent les expériences personnelles et professionnelles. L'auteur propose ici son propre parcours, elle retrace le long chemin qui l'a menée, depuis les exils obligés jusqu'au nomadisme de la vie adulte, à s'inscrire à la Société de psychanalyse de Montréal. L'inoubliable des amours perdues détermine la poursuite inlassable des rencontres. C'est cette quête, vouée à la répétition, que l'auteur poursuit dans son travail de clinicienne et dans les aspects théoriques qu'il comporte.

«L'abîme ouvert par l'événement ne saurait être mesuré.

En vérité il est impossible... de le comprendre, donc de l'intégrer à notre conscience.

Impossible de l'oublier.

Impossible aussi, quand on en parle, d'en parler, et finalement comme il n'y a rien à dire que cet événement incompréhensible, c'est la parole seule qui doit le porter, sans le dire.»

Gershom Scholem, Claudie Cachard, 1989

\begin{abstract}
«[...] je ne connais toujours pas
le nom de la femme que j'ai rencontrée de jadis à jamais dans le labyrinthe aimanté du destin

je ne suis plus revenu de cette fois ni de l'inconsolable ni de l'éternité j'ai mal d'elle que j'aime sans savoir dans l'égarement des années d'oubli.»
\end{abstract}

Gaston Miron, 2003

\section{L'appel de la formation}

a formation psychanalytique s'est imposée à moi comme un appel inflexible par un bel après-midi de printemps, alors que j'étais occupée à ratisser les feuilles mouillées encore lourdes d'hiver. Ce fut un formidable élan, et je fus pleine de certitude dans l'acquiescement et l'accomplissement de mon acte. J'allais présenter ma candidature à la Société psychanalytique de Montréal. Quelques semaines plus tard c'était chose faite.

Quand je cherche les motivations qui poussent à une telle entreprise, je vois défiler sur la longue chaîne de ma destinée, flairant déjà la brunante, la trame des 
événements creusant le lit d'un parcours de vie. Depuis la sortie de la première demeure, qui mène vers d'autres exils — qui auront été aussi bien détours en des lieux d'asile ou de véritables sanctuaires de soins — je me plais à imaginer la ligne pleine de cohérence qui porte mon nom et ma fonction, tous deux jumeaux inséparables. Je refais le chemin dans tous les sens et d'où que je reparte, j'y découvre cette voie de «formation» qui engendra l'analyste que je suis devenue.

Se pose alors la question: quand et pourquoi devient-on analyste ? Mais aussi, comment y suis-je venue ? et comment d'autres y viennent-ils, psychologue, psychiatre, philosophe ou musicien? D'où procède ce don qui fait de certains d'entre nous des voyants s'acharnant à développer l'art de la guérison — ou de se mouvoir à l'intérieur de soi et dans le monde - tout au long de notre vie ? Que penser de ce que dit Claudie Cachard sur ce métier d'analyste fait pour ne s'intéresser qu'à «soi», ou ne répondre qu'à «ses besoins» (Cachard, 1989, 17) ? Parce que d'amour en arrachement, «de train en train, de port en port, je n'ai pu oublier ton corps » (Serge Lama)... Pour explorer à répétition de nouvelles voies de recherche en vue de retrouvailles jamais achevées avec «[...] quelqu' un qui est plus moi que moi en dedans de lui. » (Gaston Miron, 2003, 66) J'ai conçu le récit de ma venue à la SPM de façon personnelle parce que le rapport que j'entretiens depuis longtemps avec la psychanalyse, cette bienveillante-malveillante, est premier et viscéral.

\section{L'avant-scène du parcours analytique}

Animée d'une passion ignorée d'elle-même, j'entrepris à la fin des années 1960 une première formation professionnelle visant à me désigner conseillère d'orientation. Dès ma sortie de l'Université Laval, je trouvai un poste à l'école secondaire Émile Nelligan située dans un quartier très défavorisé de la Ville de Montréal. Le travail d'orientation me plaisait, mais sans plus. Ce qui m'intéressait en revanche c'étaient les visites régulières des jeunes filles qui venaient non seulement discuter de leur future carrière, mais aussi déverser un peu de leur misère, lot de bien des gens de ce milieu. Je fus particulièrement interpellée par les rencontres quotidiennes d'une jeune adolescente que je nommerai $R$.

R. se présentait à mon bureau tous les jours après l'heure des classes. Elle s'assoyait d'office, visiblement assaillie par l'angoisse. Elle était tourmentée par ses antécédents, des géniteurs fantomatiques forçant son imaginaire de leurs voix bruyantes, la laissant en proie à une agitation constante. Souvent, elle se réfugiait chez l'infirmière et, là, s'enfermait dans le cabinet de toilette pour crier à tue-tête pendant des heures. Chez moi, elle venait parler avec grande parcimonie, se cachant le visage derrière son abondante chevelure. Elle se risquait, peu à peu, à poser un regard sur chacune des figures tragiques. Elle se débattait dans ce thêâtre macabre pour ne pas sombrer dans le scénario familial qui, elle le sentait, l'appelait à rejoindre les siens. Comment sortir de scène ? Comment intégrer le lieu trouble de ses origines à sa famille d'adoption, de classe aisée, dont elle était l'enfant chérie? Telle, alors, se répétait la dramatique à l'œuvre dans nos 
rencontres. La jeune exilée trouvait chez moi l'abri indispensable où se terrer pour dialoguer avec ses revenants, le temps de voir où la porteraient ses pas. Après trois années de cette consultation peu usuelle, la direction des études, avisée et inquiétée de mon intervention, m'a fait alors comprendre que je devais cesser cette pratique illégale tant du point de vue de la définition de ma tâche que de l'espace-temps offert spontanément de ma part. J'eus alors confirmation de ma vocation et j'en conclus que la pratique institutionnelle ne me permettrait pas le type d'implication qui me correspondait.

C'est au cours de ces années que je rencontrai mon premier Freud, dont l'effet constaté aura été celui d'une véritable détonation. Ce Freud m'apparut au premier abord bien sévère, voire dangereux, par ses positions sur la femme qui troublaient la jeune étudiante féministe que j'étais - rappelons qu'alors les femmes ne constituaient pas dix pour cent du contingent des étudiants en sciences humaines. J'étais fascinée par cet homme qui théorisait et pratiquait une méthode visant à faire affluer chez ses malades les contenus les plus inavouables et les plus explosifs dans l'espoir de les désactiver par leur simple exposition, à l'instar de trésors archéologiques enfouis se pulvérisant hors de leur lieu de conservation. Ce sombre Freud, animé des songes de ses nuits, me permis d'envisager notre sort commun d'humains à la vie d'âme terrifiée, ayant pour seuls témoins les mythes, les légendes et les contes, depuis la nuit des temps. Mais, de ce sous-sol hanté duquel Freud s'acharnait à vouloir faire refluer l'inconscient, je cultivais alors l'espoir tenace et la volonté ferme de me tenir éloignée. «Ne réveillez pas cette nuit les dormeurs », dit la chanson pour oublier Dachau (Aragon) (Semprun, 1994, 240)... L'attrait sublime de ces territoires excitants, pleins de bouillants sujets, finit par réveiller la dormeuse en moi.

Je m'inscrivis alors à la maîtrise en psychologie à l'Université de Montréal et je me fis engager par une firme privée de psychologues qui me fournissait une clientèle et m'assurait d'une supervision répondant aux normes requises pour être psychothérapeute ${ }^{1}$. Mais il fallut bien trouver remède à la chose tenace qui me tombait dessus et me plombait. J'entrepris alors une démarche psychothérapique en groupe restreint, qui me semblait présenter l'avantage de me faire disparaitre et, surtout, de diluer l'attention du dirigeant à mon égard. C'est ainsi qu'un peu perdue dans cette famille reconstituée j'ai pu prendre la place qui me revenait. Ma vie s'ouvrait à des émois inédits, soutenus par des liens de grande proximité et un sentiment de soi mieux assuré de sa force et de sa valeur. J'émergeais au grand jour, hors de ma famille d'origine. À cette époque, pour reprendre la formule de Jorge Semprun, «je m'enfonçais dans le silence, dans l'épuisement du désir de vivre» (Semprun, 1994, 213), bien qu'il me fallût encore naviguer entre les espaces ouverts sur des bonheurs longtemps espérés et les appels incessants des mondes intérieurs, entre cette quête insensée de l'effacement et ces voix qui en dépit de tout s'imposaient en hurlements d'urgence. 


\section{La découverte de Winnicott}

C'est au début des années quatre-vingt que je fis la deuxième rencontre déterminante de mon parcours de future analyste par l'entremise de la lecture de Jeu et réalité de Winnicott. Mon Winnicott d'alors était un homme qui s'intéressait à la mère des premiers temps, folle de son enfant, mêlant ses gestes, son souffle, sa voix et sa fatigue intense à ce petit hors d'elle, en elle, si étranger et à la fois sien — la mère-monde, la mère-sein accordée, désaccordée. J'ai retenu de cette lecture reprise maintes et maintes fois les émois du couple mère-enfant, mais, surtout, de cette mère qui donne naissance une deuxième fois à son enfant et, par là, naît d'une nouvelle vie qui n'aura de cesse de se réanimer. Comme nous le rappelle Pablo Neruda, «Para nacer he nacido», qui peut se traduire par «pour naître je suis né» ou «je ne suis né que pour naître» (Gomez Mango, 1999, 245). Cette première rencontre avec Winnicott parlait à la fois à la jeune mère que j'étais et à la clinicienne que je devenais, elle parlait à quelqu'un en moi qui se découvrait dans cet univers passionnel du maternel. La psychanalyse ainsi retrouvée depuis le maternel a été pour moi l'expérience de l'autre, si différent dès le départ et pourtant si familier. J'ai découvert que je pouvais supporter cet autre au quotidien, le tenir si près de moi, dans l'écart, pour le faire advenir à lui-même, cet autre qui me faisait absence et créait ainsi sa propre vie. Winnicott me donna les mots pour élaborer ce sujet qui sommeillait en moi, et me donna aussi l'impulsion d'aborder le divan.

«Parfois, "l'étonnant est que la lumière que nous cherchions à tâtons au plus intime de nous-mêmes, c'est du dehors qu'elle nous parvient. 'Comme si, disait Breton, je m'étais perdu et qu'on vint tout à coup me donner de mes nouvelles'. [...] Ce qui est le plus mystérieux, c'est qu'ainsi ce qui s'éprouve, de nous, habite dans le monde sans que nous l'ayons su jusque-là." ” (Gantheret, 1996, 59-60)

\section{La cure psychanalytique}

J'étais décidée à revenir aux lieux de mes origines et à affronter dans ce bureau, véritable antichambre de ma mémoire, ce que représentait pour moi la présence de l'autre. Je n'imaginais pas toutefois que ces visites quotidiennes me mèneraient depuis l'absence à mes exils. La fissure dans l'oubli — voilà comment je qualifierais ce parcours qui, du plus profond des ténèbres, tel l'œil de Caïn, poursuit son humble créature pour la rappeler à sa source. La cure analytique convoqua en moi l'événement et lui assigna un rôle qui deviendra central dans le tracé de mon parcours professionnel. La cure fut pour moi l'occasion de raconter l'histoirefiction de ma vie, faite de souvenirs et de récits brouillés. Celle-ci aurait pu se lire ainsi : il y aura désormais un avant et un après, la clarté et les ténèbres se côtoieront, mais ne se conjoindront plus - l'oubli ne sera plus l'unique compagnon de voyage. 
Pendant des années, un cri muet exigeant la présence de quelqu'un de secourable m'est fréquemment venu. Je lui cherchais des mots et me voulais une voix, mais une vie naissante souvent s'agite et ne se laisse pas saisir impunément. Cette nouvelle existence qui s'offrait à moi, je ne pouvais pas, ni ne voulais la nier, mais je ne savais pas toujours supporter la déferlante faite de bonheur et d'horreur à parts égales. J'en venais parfois à regretter mes jours d'avant, paisibles et mornes. J'aurais voulu qu'une force m'inscrive dans la mort douce de la routine familière, et que cessent les tourments provoqués par l'émergence d'une vitalité qui ne n'aurait pas dû être mienne. Toutefois, cet indicible événement me convoquait toute; il fut la faille, il fut le tiers depuis lequel l'écho de l'insondable cri a pu prendre forme et se faire chair.

Désormais, quelle langue serait mienne - quelle serait ma langue? Des années, je longeais le corridor, pour me rendre chez l'analyste, avec cette idée qui me hantait: «erreur sur la personne». Cette énigme ne cessait de m’interroger. En ce lieu s'est fait jour cette position où «je» s'adresse à «me», ou «je » se terre en son centre; où «je» observe intensément, impérieusement, le «tu». Je revins à cette posture de l'observatrice qui était mienne. Je revins à celle qui découvre, jauge, juge, se tait, réfléchit, rêve, s'affole, pleure et crie en silence : l'air calme et douce en apparence. J'emprunte les mots de Semprun : «Je suis devenu un autre pour pouvoir rester moi-même.» (Semprun, 1994, 255). Je réalisai alors que, de cette retraite, j'avais aussi été participante. Les mots, surgis d'on ne sait où, me blessaient de leur troublante vérité. Je cherchais à les éviter, mais ils me pourchassaient dans leur patiente ascension depuis le cœur jusqu'à la bouche qui les prononce et aux oreilles qui les entendent. Mystère et paradoxe de la présence et de la perte entremêlées, mais, aussi, de la rencontre et des retrouvailles. En réponse à cette occupation interne, je fus prise d'une envie irrépressible d'aller, d'un enfant à l'autre, d'un patient à l'autre, dupliquant cette nostalgie mélancolique de l'être humain qui rêve du temps de la béatitude océanique, du temps d'avant que tout se fracasse sur les événements de la vie. Je fus prise du désir de vivre et de revivre une histoire d'amour qui soit tout à la fois une nouveauté absolue et un retour à cet état rêvé de fusion déjà vécu.

\section{La psychanalyse}

Quelques années à fréquenter l'analyste réactivèrent en moi la passion pour l'homme Freud, le père de la psychanalyse, et me portèrent à la lecture passionnelle de la totalité de son œuvre. Suivirent la lecture et l'étude des principaux auteurs qui élaborèrent la pensée freudienne. Je fus poussée, insatiable, à les visiter les uns après les autres, à en discuter et, par-dessus tout, à en débattre depuis mon expérience clinique. Le retour sur les faits et gestes qui se sont imposés à moi et ont façonné ma vie, entremêlé aux lectures théoriques, échos de ces débats intérieurs, contribuèrent peu à peu à donner forme à une pensée que je pus m'approprier. Si la lectrice de toujours se sauvait dans les livres, ces lectures témoignèrent d'un début de déprise de l'événement me permettant de le penser. 
J'emprunte la question de Philippe Nemo à Lévinas : mais comment commence-ton à penser?

«Cela commence probablement par des traumatismes ou des tâtonnements auxquels on ne sait même donner une forme verbale: une séparation, une scène de violence, une brusque conscience de la monotonie du temps. C'est à la lecture des livres - pas nécessairement philosophique - que ces chaos initiaux deviennent questions et problèmes, donnant à penser.» (Lévinas, 1982,11)

C'est donc animée du désir d'en connaître davantage non plus seulement de moi, mais de la psychanalyse elle-même, que j'en suis venue à faire une demande à la Société de psychanalyse de Montréal, dans l'espoir d'y trouver une communauté d'esprit de laquelle apprendre et avec laquelle partager. Cette entreprise résulte d'un engagement depuis longtemps en action, mais exercé hors des murs de l'institution, clandestinement en quelque sorte, à l'instar du sans-papiers qui, habitant le pays depuis quelques décennies, tente finalement d'officialiser sa situation. Ma clientèle se compose de gens qui viennent à moi après avoir tenté de régler leurs difficultés par d'autres approches plus courtes et prometteuses au premier abord mais les laissant insatisfaits en définitive. Ma présence les encourage à explorer leur sujet. Déroutés, au départ, et par moments épouvantés, ils finissent par trouver une réponse à leur quête profonde d'être entendus, et prennent goût à se colleter avec leurs complications internes. J'en conclus que le tragique de notre humanité, et l'irrationnel propre de l'humain, ont besoin plus que jamais de cette écoute avec la «troisième oreille » (T. Reik) qu'offre la psychanalyse. Le monde actuel, surmédicalisé, cherche à restreindre en matière de traitement psychologique l'intervention humaine à la seule mécanique efficace, ce qui ne me semble pas être une solution permettant de dénouer les conflits psychiques importants. En effet, la psychanalyse s'est avérée, dans ma pratique clinique aussi bien que dans mon expérience de vie, la réponse la plus convaincante à l'ampleur de la force qu'engage l'événement. Elle seule, je le crois, permet de le traverser et de le comprendre pour le dire et s'en déprendre. L'institution psychanalytique, contrairement aux institutions que j'ai rencontrées à ce jour, promeut l'acquisition d'un savoir théorique constamment confronté au vif de la rencontre clinique, objet de ma recherche et creuset de la pensée psychanalytique. La psychanalyse, au plus près de ma conception, me semble ainsi permettre une formidable avancée dans un monde qui s'affiche sans peur et sans limites face aux abîmes qui bornent le quotidien de l'homme. La psychanalyse ne tente pas en cela d'éviter l'événement ni de le banaliser en proposant des solutions; elle lui donne plutôt une voix aux multiples clameurs qui, d'échos en échos, reçoivent l'accueil d'une oreille attentive. Elle se tient dans la rencontre entre deux êtres - deux espaces psychiques (Winnicott) - dans cette nécessité première d'être tenu corps et âme pour survivre 
et vivre tout à la fois. Au départ, nous sommes vie suspendue au regard de l'autre, en ce temps où sont indissociables être et être aimé. Alors l'ultime danger nous fait remarquer Gantheret, serait la perte d'être puisque :

«Nous ne voyons, par essence, que depuis les yeux de la mère. Comment alors le sujet ne vacillerait-il pas, ne basculerait-il pas, lorsque c'est la mère elle-même qui sape ce fondement en disant: de là où je suis, et donc de là où tu t'es, où tu es constitué, et au prix de ce "si" je ne te vois pas ? Ainsi la phrase fatidique prononcée par la mère n'est pas seulement expression plus ou moins directe d'un vœu de mort, ce qui ne serait que très banal et laisserait au sujet toute possibilité — sinon tout loisir ! - de se colleter avec un tel désir; elle est bien plus que cela elle est, en elle-même, acte d'anéantissement. On peut alors comprendre comment elle produit dans l'être même du sujet l'effet de sidération interne, de "trou noir" où il risque en permanence de s'engloutir. » (Gantheret, 1996, 132)

Ainsi défini, le regard de la bienveillante - la psychanalyse, l'analyste ou le parent, celui ou celle qui accompagne l'être naissant - contient tout à la fois la malveillante et son pouvoir, aussi immodéré qu'intenable. Le voyage depuis l'exil de la première demeure de chair jusqu'au nomadisme constitutif de la vie adulte exige des soins aux précautions infinies, vouées à l'imperfection puisque déjà ancrée dans le tragique. La psychanalyse, cette bienveillante-malveillante, sait que «le malheur est l'extrême de l'inattention.» (Blanchot, 1969, 178) Elle sait aussi que l'événement tragique, dans l'excès qui le caractérise, contient une richesse insoupçonnée, car c'est aussi l'événement psychique, un appel à la transformation. Les multiples surgissements qui, depuis le premier exil, nous jettent déjà dans l'affligeante séparation, nous permettent aussi la rencontre du regard amoureux et de sa présence convaincante, poussant à la poursuite du parcours périlleux. Je poursuis avec l'idée de Blanchot «[le] rapport de l'attention au malheur est le rapport que la plénitude de l'amour peut seule rétablir avec le malheureux. C'est l'amour seul, l'amour devenu l'immobilité et la perfection de l'attention qui par le regard d'autrui, ouvre une voie vers la clôture du malheur. » (Blanchot, 1969, 177178)

J'en suis venue à comprendre l'acte psychanalytique comme une injonction proférée par le patient qui arrive et dit: «REGARDE AVEC ATTENTION». Ainsi :

Le patient revient, toujours muet, ignorant de plus en plus ce qu'il est venu chercher et crie: «N'entends-tu pas la colère rageuse née de leur absence?» 
Lors de la première séance, la patiente note au passage qu'elle est angoissée. Les années suivantes, après le silence habituel, elle répond invariablement quand je m'informe d'elle: «ça va très bien » d'un ton définitif, clôturant la conversation. «Devine, ditelle, le plein obturé par le rien... Quelle route! Quelle déroute!»

D'où me vient cette impression de gêne étrange sous le regard de cette belle femme assise dans ma salle d'attente? En un fragment de seconde, je deviens sous ce regard chose détaillée, complètement dépouillée de mon sentiment d'appartenance à l'humanité. Je me dis que nous aurons une longue pente à remonter.

Quelques années à être charmée, voire excitée, par l'éloquence du discours du patient dont la voix s'élève en moi : «n'entendstu pas, là, mon désespoir de te rencontrer?» (extraits de cures)

Le patient arrive et dit regarde avec attention. Il nous convoque en ces lieux d'historicité où les images, les voix, les émois révèlent des passions secrètes engendrant par leurs forces irrépressibles des destins uniques. Nous les accompagnons, complices, dans ces marches forcées à travers les agitations et les enfoncements dans l'oubli, souvent perclus d'immobilités. Nous nous appliquons, tels des détectives, à retracer dans les bizarreries ordinaires, indignes d'attention, les mobiles qui les poussent à sauvegarder leurs tourments tels des trésors enfouis.

Pourquoi chérissons-nous la scène du tragique interne, nous, être humains, qui ne manquons pas de nous attacher à des buts ordinaires. Nous qui tentons fermement d'échapper aux forces psychiques souterraines? Comment recevoir des patients quotidiennement sans ressentir une fascination à la vue de leur affliction, tel le voyageur envoûté par la béance du Grand Canyon? Pourquoi choisir de fréquenter ces lieux d'abîmes qu'explore la psychanalyse? J'en conclus aujourd'hui que c'est pour reconstituer le pire et y échapper encore et encore; pour effrayer les jamais plus, les trop tard, les sans fin; pour maitriser l'accident majeur qui a failli me coûter la vie. Pour finalement rencontrer le regard attentif. La psychanalyse m'autorise ces voyages incessants vers le si proche, lointain. Et je pars sans cesse à la recherche de cet amour né dans l'Exil.

Je la nomme Exil, mon amour, ma très familière de cet ailleurs lointain. Je la nomme Rêve ma très présente dans cette absence sans fin. Je la nomme Ma la source prodigue de soin. 
La trace de la présence est maintenue par des histoires d'amour, d'abandon, de détresse et de retrait, de goût de mort et de désir de vivre. L'homme migrateur va de séparation en commencement. Il s'attache à ses origines. L'amour des commencements de Pontalis le lie indissociablement à tous ses actes futurs. Dans ses pérégrinations, l'homme peut avoir l'impression de s'être éloigné, peut-être même perdu, dans des contrées étrangères loin de sa terre natale, mais le poète, lui, sait que ces «voyageries » ne visent qu'à le ramener chez lui.

«J'ai mis beaucoup de temps.

J'ai fait tant de pas

pour apprendre que j'allais chez moi.» (Vigneault, 1990)

Il s'agit pour moi de tracer, retracer, partir, repartir et revenir mue par une source d'énergie inépuisable diffusée par le regard de la bienveillante. Voilà ce qui motive le périple de cette vocation insolite d'analyste. Entreprendre une énième formation, c'est ainsi poursuivre le dire à répétition, au service de la vie, telle la répétition pour symboliser ( $\mathrm{R}$. Roussillon) ou la compulsion à représenter comme nous l'a décrite de façon si éloquente, Jean Claude Rolland:

«Il en serait de la répétition ce que Freud, dans le beau texte qu'il lui consacre, dit de la négation: qu'elle est, dans le moi, la marque du refoulement, l'équivalent de Made in Germany. Ce temps de la répétition qui manifeste ainsi ses effets dans le moi par sa très subtile et impensable contrainte à penser, contrainte à repenser ses pensées, à ré-élaborer ses souvenirs, à reconstruire incessamment sa propre représentation de soi, ne serait-il pas cette cinquième saison qu'évoque J-B Pontalis? Temps insolite s'ajoutant à leur insu, aux temporalités coutumières du moi, les hantant. Et, inscrivant dans le discours préconscient, comme la brise marine porte les parfums des lointains rivages, la mélancolie des représentations inconscientes, serait-il ce qui, selon la belle expression d'André Green, désendeuille le langage ?» (Rolland, 1998, 269)

L'analyste prend forme dans l'œuvre de la vie. Sa formation s'enlace à celle-ci pour tisser, dans l'oscillation entre les événements et l'étude de leurs mouvements d'âme, la pièce à achever, jusqu'au moment, où il pourra dire: «La vie était un beau métier». (Vigneault, 1990, 83)

gisèle lacerte 290 empire longueuil (Québec) j4v 1v2 giselelacerte@hotmail.com 
Filigrane, automne 2007

\section{Note}

1. À cette époque, l'Ordre des psychologues du Québec exigeait une supervision de trois cas sur trois ans, totalisant deux cents heures de pratique. Ce parcours donnait droit au titre de psychothérapeute.

\section{Références}

Blanchot, M., 1969, L'entretien infini, Paris, Gallimard.

Gantheret, F., 1996, Moi, Monde, Mots, Paris, Gallimard.

Cachard, C., 2006, Les gardiens du silence, Paris, Des femmes.

Gomez Mango, E., 1999, «Fragments vers le natal», Le fait de l'analyse, nº 6, 243-267.

Levinas, E., 1982, Éthique et infini, Paris, Fayard.

Miron, G., 2003, Poèmes épars, Québec, l'Hxagone.

Rolland, J-C., 1998, Guérir du mal d'aimer, Paris, Gallimard.

Semprun, J., 1994, L'écriture ou la vie, Paris, Gallimard.

Vigneault, G., 1990, Chemin faisant. Cent et une chansons du Québec, auvidis-le Nordet. 